\title{
Clinical and Radiologic Findings of Acute Necrotizing Encephalopathy in Young Adults
}

\author{
(D) H.A. Vanjare, (D) B.T. Selvi, (D). Karuppusami, (D) A. Manesh, (D) K. Gunasekaran, (D) A.T. Prabhakar, (D) P. Mannam, and (D) A. Jasper
}

\begin{abstract}
SUMMARY: Acute necrotizing encephalopathy after an acute febrile illness, although initially described exclusively in the pediatric age group, has been recently shown to have an adult onset as well. In this study, we describe 10 patients (16years of age or older) with acute necrotizing encephalopathy. In our study, bilateral thalamic involvement with the trilaminar pattern of diffusion restriction on MR imaging was the predominant finding seen in all of the patients reviewed. Ancillary findings of cerebral white matter, brain stem, and cerebellum involvement with sparing of the basal ganglia were also noted. A poorer outcome was observed in patients with a higher degree of thalamic involvement. The cause of an underlying infection was identified in 4 patients (dengue in 3 and influenza in 1). Overall, a sizeable portion of young adults with acute necrotizing encephalopathy have shown a poorer outcome, with dengue being an important underlying trigger in an endemic region.
\end{abstract}

ABBREVIATION: ANE = acute necrotizing encephalopathy

A cute necrotizing encephalopathy (ANE) is a parainfectious, pauciinflammatory disorder predominantly reported among children younger than 5 years of age. The diagnosis is based on the typical clinical presentation, imaging findings, and exclusion of other mimicking conditions. ${ }^{1,2}$ Although the pathophysiology of ANE is not completely understood, it is considered to be immunemediated after a viral infection. ${ }^{3-5}$ A lack of inflammatory changes in ANE distinguishes it from other necrotizing encephalitides.

Hypercytokinemia, also known as "cytokine storm," 6,7 is the most widely accepted theory that described the triggers for the development of ANE in susceptible hosts and is characterized by elevated serum interleukin (IL)-6, $-10,-15$, and $-1 \beta$; tumor necrosis factor- $\alpha$ (TNF- $\alpha$ ); and interferon- $\gamma \cdot{ }^{5-8}$ Whereas neurotoxicity is associated with a higher concentration of IL-6, TNF- $\alpha$ is associated with endothelial damage involving the intracranial vessels. ${ }^{8}$ Autopsy and histopathology of a previously reported patient showed coagulative necrosis accompanied by hemorrhages around the small vessels in the involved regions of the brain. Lymphocytic and neutrophilic infiltrates were not observed, which is suggestive

Received May 24, 2020; accepted after revision July 15

From the Christian Medical College and Hospital, Tamil Nadu, India.

Please address correspondence to Dr. Harshad Arvind Vanjare, Dept. of Radiology, Christian Medical College and Hospital, Ida Scudder Rd, Vellore 632004, Tamil Nadu, India; e-mail: harshadcmc@cmcvellore.ac.in

Indicates article with supplemental on-line tables.

Indicates article with supplemental on-line photos.

http://dx.doi.org/10.3174/ajnr.A6803 of a lack of cellular inflammatory response. ${ }^{9}$ The lack of inflammatory cell infiltration might suggest a distant, spin-off effect because of uncontrolled inflammation secondary to the primary inciting event, commonly a viral infection. A missense mutation in the gene encoding the nuclear pore protein Ran binding protein 2 is found to increase an individual's susceptibility for ANE. ${ }^{10,11}$ The mechanistic understanding between this mutation and the pathway leading to ANE is unknown; however, the mutation may affect energy production and lipid peroxidation within the cells along with altering the mechanism for viral entry, cytokine signaling, antigen presentation, immune response, and blood-brain barrier maintenance. $^{10}$

Case reports have been published from around the world and those from East Asia in the late 1990s described symmetric brain lesions with bilateral thalamic involvement. Because most of the published literature involves the pediatric population, it is often labeled as ANE of childhood. The literature on older patients with ANE is sparse with only 30 cases published to date (PubMed and Google Scholar search without language restriction and age cutoff of 16 years). Hence, there are inadequate data to describe the pattern of brain parenchymal involvement among older patients and its possible correlation to final or long-term outcomes.

In this single-center retrospective study from a medical college hospital in South India, all patients (16years of age and older) with clinical and radiologic findings suggestive of ANE were analyzed, and their clinical, imaging, laboratory findings, and outcomes were documented. 

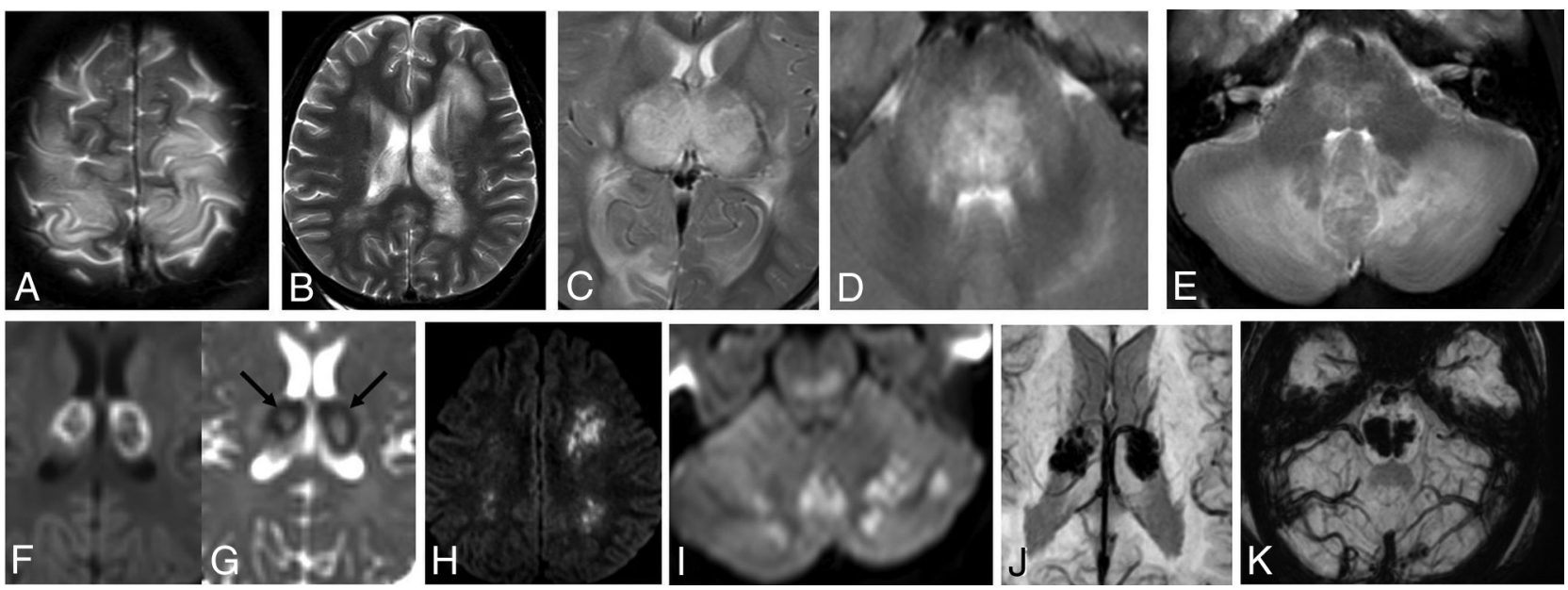

FIGURE. A-E, T2-weighted axial sections demonstrating the involvement of cortical gray matter, white matter, thalami, brain stem, and cerebellar hemispheres. F-I, DWI axial sections demonstrating diffusion restriction in thalami, cerebral, and cerebellar white matter. Trilaminar pattern on $A D C(G)$ as a low signal ring (arrows) with central and peripheral hyperintensity.J and $K$, SWI demonstrating microhemorrhages in thalami and brain stem.

\section{CASE SERIES}

The study was approved by the institutional review board. Patients were identified retrospectively by using the radiology database and hospital information system (from 2002 to 2019) based on the features proposed by Mizuguchi and coworkers, ${ }^{1,2}$ which are as follows: 1) acute encephalopathy after a febrile viral disease and rapid deterioration in the level of consciousness; 2) CT or MR imaging findings for symmetric, multifocal brain lesions, including bilateral thalami; and 3) exclusion of other resembling diseases such as cerebral venous thrombosis, hypoxicischemic injury, and metabolic etiologies.

Patients younger than 16 years of age were excluded. Clinical and laboratory parameters were documented. A good outcome was defined as absent or mild to moderate neurologic deficits on follow-up, and a poor outcome was defined as severe neurologic deficits on follow-up or death during the hospital stay.

\section{Technical Information}

MR imaging scans were performed on a 1.5T (Magnetom Avanto, Siemens) or a 3T (Intera Achieva, Philips Medical Systems) scanner, and the CT scan was performed on a 64-section scanner (Discovery $750 \mathrm{HD}$, GE Healthcare).

Ten patients with a mean age of 23.8 (SD, 5.9; median, 23.5; range, 17-32) years were included in the study. There were 8 men and 2 women. All the patients were of South Asian (Indian) descent with no premorbid illness or medical condition or family history of similar illness.

\section{Clinical and Laboratory Parameters}

Patients presented with fever, altered sensorium, vomiting, and seizures, clinically raising the suspicion of encephalitis (On-line Table 1). The onset of symptoms was associated with the development of fever in all patients followed by other symptoms. The mean duration between the onset of fever and imaging was 3.6 days (SD, 1.07; median, 4 days; range, 2-5 days). On blood investigations, transaminitis was seen in 4 patients. CSF analysis showed elevated proteins in all 5 patients in whom the analysis was performed. Other parameters assessed were liver function tests, including bleeding parameters and renal function. None of the patients were tested for elevated serum cytokine levels.

\section{Viral Infections and Medical Management}

Of the 10 patients, 3 were positive for dengue and 1 for HIN1 influenza (On-line Table 2). The patients were managed conservatively with the use of antivirals, immunosuppression, and supportive care.

\section{Imaging Findings}

Nine patients underwent MR imaging, and 1 had CT (On-line Table 3 and Figure). The brain lesions were seen as areas of T2weighted hyperintensities associated with swelling with corresponding T1-weighted hypointensities on MR imaging scans, which were hypoattenuated on CT. Cerebral white matter involvement assessed on T2-weighted imaging was seen in 8 (89\%) patients with involvement of subcortical white matter in 6 patients, deep white matter in 4 patients, and periventricular white matter in 3 patients. Cortical gray matter involvement was seen in $60 \%$, brain stem involvement in $80 \%$, and cerebellar hemisphere involvement in $100 \%$ of the patients. Thalamic involvement being a part of the diagnostic criteria was seen in all of the patients. Based on visual assessment, thalamic involvement was divided into $<50 \%$, $>50 \%$ but not nearcomplete involvement, and near-complete involvement. All the patients had $>50 \%$ involvement of the thalami. The basal ganglia involvement was not seen in any patient.

Of the 9 patients in whom an MR imaging scan was performed, diffusion restriction involving the white matter was seen in $44 \%$ of patients, gray matter involvement in $56 \%$, brain stem involvement in $78 \%$, and cerebellum involvement in $100 \%$ of patients. Trilaminar pattern of diffusion restriction, which is seen as a ring of low signal involving the thalamus on an apparent diffusion coefficient map with central and peripheral hyperintensity (Fig 1G), was observed in $100 \%$ of patients.

No significant postcontrast enhancement was seen in the 6 postcontrast MR imaging studies performed. Multiple foci of microhemorrhages, seen as blooming on a susceptibility-weighted 
sequence involving the thalami, brain stem, and cerebellar hemispheres, were noted in all $9 \mathrm{MR}$ imaging scans. Cavitation (defined as a cystic area on a T2-weighted sequence and hypointense area on a T1-weighted sequence or fluid density on CT scan) was identified in 1 case with bithalamic involvement (On-line Fig 1A).

\section{Outcome}

Six patients with an average follow-up of 21 (SD, 29.6; median, 6; range, 1-76) months had good outcomes (On-line Table 4). Four patients had mild motor deficits, and 2 patients had developed mood changes with aggressive behavior. MR imaging was available in 2 patients at 5 and 31 months of follow-up (On-line Figs 1 and Fig 2); both showed residual gliotic changes in the previously involved parenchyma with resolution of diffusion restriction and edema. Four patients died during their hospital stay.

\section{DISCUSSION}

ANE is a rare, albeit severe, parainfectious disorder predominantly seen in the pediatric age group and rarely reported in older patients. The clinical course in pediatric patients is divided into an initial prodromal stage, which is associated with fever, vomiting, and cough. This is followed by the acute encephalopathy stage associated with disturbance in consciousness and seizures, finally progressing to a prolonged recovery stage when few recover completely, with residual neurologic deficits in most of the survivors. ${ }^{8}$

Most of the patients described in the literature have a preceding viral infection that triggers an excessive immune response in susceptible hosts. Most of the published adult patients with ANE describe an influenza infection as the initial trigger. A recent case report has shown COVID-19 infection as an initial trigger for adult onset of ANE. ${ }^{12}$ Dengue, as an inciting trigger for older patients with ANE, has not been described previously. Dengue was identified in 3 of our patients. In the pediatric population, ANE has been described with viral infections such as influenza, parainfluenza, herpesvirus 6 and 7, herpes simplex virus, measles, rubella, varicella, coxsackie A9, rotavirus, reovirus, and dengue. $^{8,13}$ Most of the data published on ANE are from the east Asian regions such as Japan, Korea, and Taiwan, where dengue infection is uncommon. Our case series therefore highlights the importance of dengue as a cause of ANE among young adults in endemic regions such as India.

Typical imaging findings in pediatric ANE include fairly symmetric abnormalities in bilateral thalami, cerebral white matter, brain stem, and cerebellar hemispheres. ${ }^{1-3,14-17}$ The pediatric case series by Kim et al, ${ }^{18}$ published in 2004 with 14 patients, showed the involvement of the internal capsule and deep white matter in $50 \%$ and $21 \%$, respectively. Another case series published by Wong et al, ${ }^{19}$ published in 2006 with 12 patients, showed a 67\% involvement of the cerebral matter with no distinction made between subcortical, deep, and periventricular white matter. In our case series, the white matter was involved in $89 \%$ of patients, which is more frequent than that seen in the pediatric age group. On the other hand, our studies showed a $100 \%$ involvement of the cerebellum compared with $14 \%$ and $33 \%$ in the aforementioned pediatric ANE studies, respectively. Brain stem involvement was seen in $86 \%$ and $83 \%$ in the pediatric ANE case series, respectively, which is comparable to our case series with $80 \%$ involvement.
Parenchymal hemorrhage was seen in $36 \%$ and $42 \%$ in the pediatric ANE series; this rate was $90 \%$ in our series. This difference in the degree of parenchymal hemorrhage may be related to the use of the susceptibility-weighted sequence in all of our patients in whom hemorrhage was identified. There is a lack of published data describing diffusion restriction in different brain structures in ANE. In our patients, we found the involvement of posterior and central structures such as the cerebellum, brain stem, and thalami to be more common compared with the anterior structures such as the cerebral white matter and cortical gray matter.

Bithalamic involvement can be seen in multiple disorders such as West Nile encephalitis, Japanese encephalitis, Creutzfeldt-Jakob disease, Wernicke encephalopathy, acute disseminated encephalomyelitis, and thrombosis of the internal cerebral veins or straight sinus, among others. ${ }^{20}$ Clinical presentation in patients with encephalitis can be very similar to that seen with ANE and can have significant thalamic involvement. However, a trilaminar pattern of diffusion restriction has not been described in patients with encephalitis. In Creutzfeldt-Jakob disease and Wernicke encephalopathy, the medial aspects of thalami are more involved. Acute disseminated encephalomyelitis is characterized by oval to round white matter demyelinating lesions, which may show an incomplete ring of enhancement, and thalami are not commonly involved, thereby distinguishing these conditions from ANE. Thrombosis of internal cerebral veins or the straight sinus can be identified by carefully examining T2-weighted coronal sections of the brain and postcontrast MR venograms.

Dengue is known to involve the brain. One study described the involvement of the thalami in one-fourth of the 35 patients presenting with dengue infection and neurologic symptoms. ${ }^{21}$ Both encephalopathic and encephalitic presentation with bithalamic involvement in patients with dengue is described in the literature. The clinical distinction between these 2 is not possible. Encephalitis is often associated with CSF pleocytosis, which helps to distinguish it from encephalopathy, in which the CSF does not show an elevated cell count.

The presence of hemorrhage and cavitation are shown to correlate with poor outcomes in pediatric patients with ANE. ${ }^{19}$ Similar data are not available for adult patients. In our series, microhemorrhages were seen on the susceptibility-weighted sequence in all 9 patients for whom MR imaging was performed with the involvement of central and posterior structures such as the thalami, brain stem tegmentum, and cerebellar hemispheres. Cavitation involving bilateral thalami was seen in 1 case with a good outcome.

Bilateral thalamic involvement is essential for the diagnosis of ANE; however, the degree of thalamic involvement and its correlation with the outcome has not been described in the literature. In our series, none of the patients had $<50 \%$ of thalamic involvement. Three patients with $>50 \%$ thalamic involvement (but less than near-complete involvement) were seen, all with good outcomes. Near-complete thalamic involvement was seen in the remaining 7; 4 had poor outcomes, and the other 3 fared well. Trilaminar pattern of diffusion restriction on the ADC sequence is considered to be specific for $\mathrm{ANE}^{8}$ and was corroborated in all our patients with MR imaging, thus lending support to this imaging finding as an easy-to-identify imaging maker. 
Brain stem lesion as a predictor for a poor outcome is controversial; however, 2 scoring systems have shown a correlation between brain stem lesions and poor outcome in pediatric patients. ${ }^{19,22}$ In our case series, $80 \%$ of the patients had brain stem involvement (4 patients in the good outcome group and 4 patients in the poor outcome group).

Laboratory predictors for outcome have been inadequately assessed in adult patients. Normal or a mild increase in CSF protein in pediatric patients with ANE is associated with better clinical outcomes compared with those with elevated levels. ${ }^{18,23}$ In our series, CSF analysis was available in 5 patients, all of whom had elevated levels of proteins and had good outcomes. This difference between the young adult and pediatric patients with ANE may be related to a more mature immune response with age.

There is no standardized protocol of management and treatment of ANE, so these are often tailored on a case-by-case basis and local expertise. Medical management mainly remains supportive with some studies showing benefits with the use of antivirals, immunoglobulins, and steroids. ${ }^{24,25}$ The number of patients in our study were small with limited data on specifics of management to make any definitive observation. There is recent literature that shows the benefit of IL- 6 blockade in the management of cytokine storm. ${ }^{26}$ Similar intervention may benefit patients with ANE if the cytokine storm truly plays an important role in its pathogenesis. Sixty percent of patients in our series had good outcomes; however, these patients did not improve to their premorbid state and had residual motor deficits or mood changes. Despite medical advances, the poor outcome in ANE remains high with a $40 \%$ mortality rate seen in our case series.

\section{CONCLUSIONS}

ANE in young adults is a rare immune-mediated disorder commonly involving the thalami, brain stem, and cerebellum. The characteristic trilaminar pattern of diffusion restriction on MR imaging, described in the literature, was seen in all of our patients. Coincidentally, in our study, we found that cerebellar involvement and parenchymal microhemorrhages were observed at a higher frequency than that which is reported in the literature for pediatric patients with ANE. The involvement of vital structures such as the thalami, brain stem, and cerebellum may explain the long-term deficits seen in survivors. Long-term sequelae include both motor deficits and mood disorders with treatment remaining largely supportive.

\section{ACKNOWLEDGMENT}

The authors thank Dr. Carl Prem Trevor Colaco, Department of Radiology, Christian Medical College, Vellore, India, for English language support during manuscript preparation.

DISCLOSURES: Pavithra Mannam—UNRELATED: Employment: Christian Medical College and Hospital.

\section{REFERENCES:}

1. Mizuguchi M, Abe J, Mikkaichi K, et al. Acute necrotising encephalopathy of childhood: a new syndrome presenting with multifocal, symmetric brain lesions. J Neurol Neurosurg Psychiatry 1995;58:55561 CrossRef Medline

2. Mizuguchi M. Acute necrotizing encephalopathy of childhood: a novel form of acute encephalopathy prevalent in Japan and Taiwan. Brain Dev 1997;19:81-92 CrossRef Medline

3. Mizuguchi M, Yamanouchi H, Ichiyama T, et al. Acute encephalopathy associated with influenza and other viral infections. Acta Neurol Scand Suppl 2007;186:45-56 CrossRef Medline

4. Ito $\mathrm{Y}$, Ichiyama $\mathrm{T}$, Kimura $\mathrm{H}$, et al. Detection of influenza virus RNA by reverse transcription-PCR and proinflammatory cytokines in influenza-virus-associated encephalopathy. $J$ Med Virol 1999;58:420-25 CrossRef Medline

5. Ichiyama T, Endo S, Kaneko M, et al. Serum cytokine concentrations of influenza-associated acute necrotizing encephalopathy. Pediatr Int 2003;45:734-36 CrossRef Medline

6. Wang HS, Huang SC. Acute necrotizing encephalopathy of childhood. Chang Gung Med J 2001;24:1-10 Medline

7. Lim HY, Ho VPY, Lim TCC, et al. Serial outcomes in acute necrotising encephalopathy of childhood: a medium and long term study. Brain Dev 2016;38:928-36 CrossRef Medline

8. Lin Y-Y, Lee K-Y, Ro L-S, et al. Clinical and cytokine profile of adult acute necrotizing encephalopathy. Biomed J 2019;42:178-86 CrossRef Medline

9. Kansagra SM, Gallentine WB. Cytokine storm of acute necrotizing encephalopathy. Pediatr Neurol 2011;45:400-02 CrossRef Medline

10. Wu X, Wu W, Pan W, et al. Acute necrotizing encephalopathy: an underrecognized clinicoradiologic disorder. Mediators Inflamm 2015;2015:e792578 CrossRef Medline

11. Ishii N, Mochizuki H, Moriguchi-Goto S, et al. An autopsy case of elderly-onset acute necrotizing encephalopathy secondary to influenza. J Neurol Sci 2015;354:129-30 CrossRef Medline

12. Neilson $\mathrm{DE}$, Adams MD, Orr CMD, et al. Infection-triggered familial or recurrent cases of acute necrotizing encephalopathy caused by mutations in a component of the nuclear pore, RANBP2. Am J Hum Genet 2009;84:44-51 CrossRef Medline

13. Marco EJ, Anderson JE, Neilson DE, et al. Acute necrotizing encephalopathy in 3 brothers. Pediatrics 2010;125:e693-98 CrossRef Medline

14. Poyiadji N, Shahin G, Noujaim D, et al. COVID-19-associated acute hemorrhagic necrotizing encephalopathy: CT and MRI features. Radiology 2020;296:E119-20 CrossRef Medline

15. Abbas Q, Jafri SK, Ishaque S, et al. Acute necrotizing encephalopathy of childhood secondary to dengue infection: a case report from Pakistan. J Pediatr Neurosci 2017;12:165-67 CrossRef Medline

16. Toovey S. Influenza-associated central nervous system dysfunction: a literature review. Travel Med Infect Dis 2008;6:114-24 CrossRef Medline

17. Britton PN, Dale RC, Blyth CC, ACE study investigators and PAEDS network, et al. Influenza-associated encephalitis/encephalopathy identified by the Australian Childhood Encephalitis Study 2013-2015. Pediatr Infect Dis J 2017;36:1021-26 CrossRef Medline

18. Kim JH, Kim I-O, Lim MK, et al. Acute necrotizing encephalopathy in Korean infants and children: imaging findings and diverse clinical outcome. Korean J Radiol 2004;5:171-77 CrossRef Medline

19. Wong AM, Simon EM, Zimmerman RA, et al. Acute necrotizing encephalopathy of childhood: correlation of MR findings and clinical outcome. AJNR Am J Neuroradiol 2006;27:1919-23 Medline

20. Smith AB, Smirniotopoulos JG, Rushing EJ, et al. Bilateral thalamic lesions. AJNR Am J Roentgenol 2009;192:W53-62 CrossRef Medline 
21. Vanjare HA, Mannam P, Mishra AK, et al. Brain imaging in cases with positive serology for dengue with neurologic symptoms: a clinicoradiologic correlation. AJNR Am J Neuroradiol 2018;39:699703 CrossRef Medline

22. Yamamoto H, Okumura A, Natsume J, et al. A severity score for acute necrotizing encephalopathy. Brain Dev 2015;37:322-27 CrossRef Medline

23. Seo H-E, Hwang S-K, Choe $\mathrm{BH}$, et al. Clinical spectrum and prognostic factors of acute necrotizing encephalopathy in children. $J$ Korean Med Sci 2010;25:449-53 CrossRef Medline
24. Okumura A, Mizuguchi M, Kidokoro H, et al. Outcome of acute necrotizing encephalopathy in relation to treatment with corticosteroids and gammaglobulin. Brain Dev 2009;31:221-27 CrossRef Medline

25. Alsolami A, Shiley K. Successful treatment of influenza-associated acute necrotizing encephalitis in an adult using high-dose oseltamivir and methylprednisolone: case report and literature review. Open Forum Infect Dis 2017;4:ofx145

26. Tanaka T, Narazaki M, Kishimoto T. Immunotherapeutic implications of IL-6 blockade for cytokine storm. Immunotherapy 2016;8:959-70 CrossRef Medline 